\title{
Avaliação do sistema de controle de alimentos através de uma abordagem que considera os stakeholders nas Ilhas Maurício
}

DOI: $10.3395 /$ reciis.v2i2.141pt

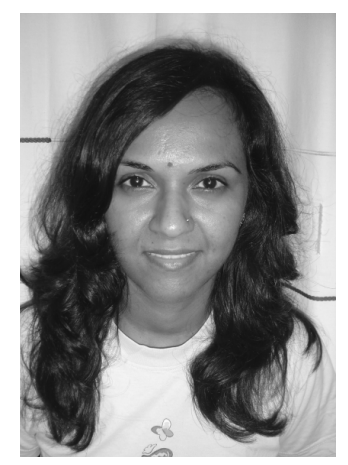

\section{Shalini A.}

\section{Neeliah}

Faculdade de Agricultura, Universidade de Maurício Réduit, Maurício san.ahiscons@gmail.com

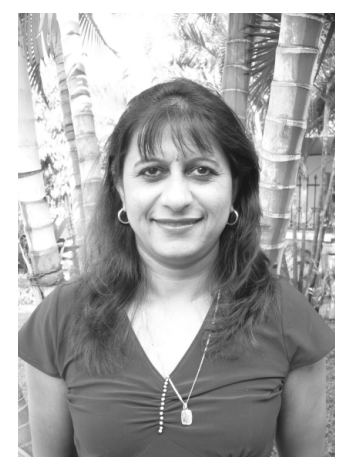

\section{Goburdhun}

Faculdade de Agricultura, Universidade de Maurício, Réduit, Maurício daya@uom.ac.mu

\section{Harris Neeliah}

Departamento de Economia Agrícola e de Alimentos, Universidade de Reading, PO Box 237, Reading RG6 6AR, Reino Unido h.neeliah@rdg.ac.uk Bolsa (taxas da universidade) financiada pela Universidade de Maurício (2002-2007).

\section{Resumo}

Os governos têm a responsabilidade de assegurar o controle de alimentos e atender aos requisitos da Organização Mundial do Comércio. Os sistemas de controle de alimentos precisam ser avaliados continuamente para melhorar e alcançar um patamar seguro. Este trabalho avalia a utilidade de uma análise dos stakeholders e o uso de um índice para esta avaliação no contexto de um país em desenvolvimento, que é a Ilha Maurício (também chamada Maurício). A metodologia aplicada compreendeu uma revisão da literatura e uma análise das partes interessadas com base em um questionário. Em média, as partes interessadas acreditavam que os componentes do sistema de controle de alimentos e a conformidade da indústria de alimentos eram adequados. A resposta dos participantes dos órgãos governamentais foi, porém, diferente da resposta dos representantes de órgãos não-governamentais para administração, execução, mecanismo institucional para consulta e estabelecimento de política sobre padrões nacionais de controle de alimentos e regulamentos e oportunidades para tornar as opiniões conhecidas pelo Codex através do Governo. Estas poderiam indicar áreas-problema. Com relação ao índice planejado por avaliar um sistema de controle de alimentos (food control system - FCS), Maurício foi classificado como um país com um FCS satisfatório. É interessante ver como estes resultados correspondem aos estudos anteriores, embora seja plenamente reconhecido que as abordagens metodológicas e os prazos diferem. A análise dos stakeholders, então, uma comprovação da condição do Sistema de Controle de Alimentos de Maurício (Mauritian food control system - MFCS) permitiu uma completaavaliação do sistema global. Dado que nenhum trabalho foi anteriormente conduzido no sentido de desenvolver um índice de controle de alimentos e categorizar um sistema adequado, outra pesquisa será desenvolvida para validar esta metodologia. Com base na análise dos stakeholders, recomenda-se que o monitoramento do FCS seja melhorado e os stakeholders redefinidos. A interação entre os órgãos reguladores e não-governamentais deve, também, ser melhorada.

\section{Palavras-chave}

sistema de controle de alimentos; análise de stakeholders; controle de alimentos; índice; Ilhas Maurício 


\section{Introdução}

O Governo de cada país é responsável pelo bemestar comum da sociedade, e para isso tem que assegurar a segurança dos alimentos. Portanto, os sistemas nacionais de controle de alimentos (NFCS) foram estabelecidos para lidar com as ameaças de um abastecimento de alimentos inseguro e são equipados para proteger a saúde dos consumidores (Kenny 1996; Bruno 1996; Boutriff \& Bessy 1999; Neeliah \& Goburdhun 2007; Nguz 2007). Muitos países atualmente estão no processo de estabelecer um sistema de controle de alimentos (FCS) ou fortalecendo os já existentes. Mas a eficácia dos NFCS é frequentemente questionada (FAO 2006). De acordo com Rees e Watson (2000), a avaliação é uma fase necessária no gerenciamento de projeto e os resultados de uma avaliação podem levar ao fortalecimento de um sistema de controle de alimentos. A avaliação contínua é, por conseguinte, sine qua non para avaliar a eficácia dos NFCS e indicar as áreas que requerem melhoria. Este exercício é importante para manter a segurança do sistema. Maurício testemunhou um rápido crescimento econômico desde o início da década de 1980 (Banco Mundial 2007) para alcançar PIB/capita de US\$5,059 (cinco mil e cinquenta e nove dólares americanos) em 2005 (UNDP 2007). Este crescimento levou a um aumento na renda da classe média, resultando em mudanças fundamentais nos padrões de consumo de alimentos e demanda pela segurança dos mesmos. Maurício tem um sistema operacional de FCS para assegurar a proteção da saúde dos consumidores. Em 2000, o FCS foi revisado com a introdução de uma moderna legislação, a Lei de Alimentos (MOH 1998). O objetivo deste trabalho é avaliar o FCS existente usando uma abordagem inovadora. Para isso, foi usada uma metodologia ainda não experimentada para avaliar um FCS. Ela se baseia em uma análise das partes interessadas, pela qual os atores-chave nos NFCS são interrogados sobre o FCS usando um questionário semiestruturado. Suas respostas são, então, usadas para planejar um índice de controle de alimentos. Este método é, discutivelmente, mais apropriado para um país em desenvolvimento, onde os indicadores de segurança de alimentos não são necessariamente e sistematicamente coletados.

\section{Revisão da literatura}

\section{Componentes do sistema mauriciano de controle de alimentos}

Os NFCS em Maurício compreendem quatro componentes principais: a legislação de alimentos, a administração, a execução e os órgãos de apoio. Vytelingum (2003) descreveu os componentes do Sistema Mauriciano de Controle de Alimentos (MFCS) em detalhes. As seções a seguir apresentam suas características de destaque.

\section{Legislação de alimentos em Maurício}

A primeira Lei relacionada diretamente ao controle de alimentos em Maurício, a Lei de Alimentos e Medicamentos (1940), foi criada de acordo com a regra britânica. Ela incorporou o princípio de proteção de saúde e aquele de prevenção da exploração do consumidor. Ela não teve qualquer objetivo definido oficialmente, mas continha determinados elementos básicos de uma lei de alimentos, compreendendo uma definição para alimentos, a competência para a implementação da lei, os poderes dos funcionários públicos e as penalidades. Considerava-se um crime, de acordo com a Lei, a adição ou subtração de qualquer substância de forma que o alimento se tornasse perigoso à saúde assim como vender qualquer alimento que não fosse da natureza, substância ou qualidade exigida.

A Lei de Alimentos e Medicamentos e seus regulamentos foram provavelmente apropriados para a década de 1950, mas se tornaram antiquados e incapazes de enfrentar as principais mudanças tecnológicas que ocorreram no sistema mauriciano de alimentos. Além disso, os regulamentos não eram abrangentes. As penalidades aplicadas por qualquer crime cometido sob a mesma montavam a um valor entre $\mathrm{Rs}^{1}$ 500-1000, com prisão, que não ultrapassava três meses. Estas penalidades para os contraventores eram absurdamente baixas e não representavam um desincentivo, especialmente para um assunto de interesse de saúde pública. A lei, portanto, não era adequada para garantir o funcionamento adequado do MFCS. Em 1998, houve uma revisão da legislação principal que levou ao desenvolvimento da Lei de Alimentos. Foi dada uma maior ênfase sobre a modernização da lei através da prescrição de novos padrões e consolidação da mesma ao incorporar todas as seções que são exigidas para uma lei moderna. A Lei de Alimentos, baseada na Lei de Segurança de Alimentos (1990) do Reino Unido, está em vigor desde $1^{\circ}$ de janeiro de 2000 . A nova lei determina que a "importação, preparação, fornecimento de alimentos que não apresentem qualidade negociável são um crime." É também crime a “importação, preparação, distribuição e venda de qualquer alimento que seja venenoso, que contenha matérias estranhas, que esteja adulterado ou que seja impróprio para o consumo humano". A nova lei também fortalece o poder de entrada em instalações de alimentos por parte dos funcionários públicos autorizados e permite o confisco de alimentos para análise (MOH 1998) e procedimentos de execução mais detalhados, que variam de notificações para melhoria a ordens de proibição de emergência. As penalidades infligidas variam de uma multa não inferior a MRU² $2.000 \mathrm{e}$ à prisão máxima de dois anos. Além disso, o Ministro da Saúde pode criar regulamentos que considere necessário para melhorar/incrementar a Lei de Alimentos. O escopo da Lei é amplo: com uma definição mais abrangente para "alimentos" 3 e uma variedade maior de atividades ou negócios alimentícios. Os regulamentos foram feitos baseados em normas internacionais da Codex $(\mathrm{MOH}$ 1998), após consultas mantidas entre os departamentos de governo, como a Unidade de Nutrição do $\mathrm{MOH}$ e o Ministério da Agroindústria e Pesca (MOA). 
Determinados problemas práticos surgiram quando a Lei de Alimentos de 1998 entrou em vigor em 2000. Estes ficaram evidentes em vários artigos da imprensa (Ramsamy 2001), os quais se relacionavam à falta de consulta aos stakeholders durante a fase de redação da lei e clamor dos fornecedores de alimentos de rua com relação às novas disposições para higiene. Outra reclamação era que havia muito pouco tempo para conformidade. Estes problemas surgiram porque a legislação de alimentos de Maurício foi adaptada da Lei Britânica de Segurança de Alimentos de 1990 e, portanto, não era totalmente aplicável ao contexto mauriciano. Além disso, Maurício não capturou todos os aspectos positivos da lei britânica, como o conceito de "devida diligência" ("due diligence").

\section{Administração e execução}

No nível de governo central, o $\mathrm{MOH}$ é responsável pelo controle de alimentos em Maurício (Vytelingum et al. 2000). A Divisão de Inspetoria de Saúde é uma filial subordinada ao $\mathrm{MOH}$ e é responsável por fazer cumprir a Lei de Alimentos de 1998. Por conveniência administrativa, Maurício foi dividido em cinco regiões nas quais existem Escritórios de Saúde. Cada escritório é administrado por um Inspetor de Saúde Principal, que é responsável por planejar e dirigir o controle de segurança de alimentos naquela determinada região. Os inspetores de saúde executam várias tarefas como a inspeção de instalações para garantir a conformidade com as leis da saúde pública, incluindo a ambiental, saúde ocupacional, higiene industrial, alimentos, comércio e indústrias; a implementação do programa de educação de saúde; a emissão da liberação para instalações como lojas de alimentos, açougues, navios e aeronaves em conformidade com a legislação de saúde pública e instauração de processo para contravenções relacionadas a higiene e alimentos (MOH 1993).

\section{Os serviços analíticos}

A Divisão de Análise do Governo e a Divisão Patológica do Laboratório Central são os principais laboratórios que analisam amostras de alimentos coletadas pela estrutura da Inspetoria de Saúde ou submetidas pela indústria alimentícia. A Divisão de Anállise realiza as análises químicas em alimentos, enquanto o Laboratório Central lida com o exame microbiológico dos alimentos e da água. Estes dois laboratórios são os únicos serviços analíticos reconhecidos de acordo com a Lei de Alimentos.

\section{Outros ministérios de apoio}

O Ministério do Governo Local (MLG) exerce o controle sobre as atividades das pessoas em uma dada localidade através dos Conselhos Distritais e Conselhos Municipais. Cada um tem um Departamento de Saúde que emite e renova licenças de desenvolvimento e licenças para instalações alimentícias mediante a liberação do $\mathrm{MOH}$. As autoridades locais também estão comprometidas na inspeção de mercados (MLG
2008), onde eles têm o dever de assegurar a qualidade sanitária dos alimentos vendidos. Os inspetores de saúde não são funcionários públicos autorizados, conforme a Lei de Alimentos de 1998, mas eles derivam seus poderes de intervenção da Lei do Governo Municipal de 2003.

O MOA tem vários departamentos que lidam diretamente ou indiretamente com o controle de alimentos. As Divisões de Química de Laticínios e de Química Agrícola conduzem a análise de diversos ítens de alimentos, incluindo a produção agrícola primária (DCD 2006; ACD 2008). A Divisão de Serviços Veterinários emite licenças de importação para produtos de origem animal. Os exportadores de produtos animais dependem da ajuda analítica e técnica dos Serviços Veterinários porque a União Européia a reconhece como seu agente (DVS 2008). Os Serviços Veterinários também estão envolvidos na inspeção dos animais de criação, na emissão de licenças veterinárias para exportação e na supervisão de processos industriais com relação a produtos veterinários para exportações (animal, peixe). Um laboratório novo, o Laboratório de Tecnologia de Alimentos, foi criado sob a égide do MOA para lidar principalmente com produtos alimentícios destinados à exportação. Ele será credenciado para a ISO 17025 para atender as exigências da União Européia.

\section{Organizações do Consumidor e Associações de Comércio}

Duas dessas organizações são conhecidas localmente: O Instituto de Defesa do Consumidor (ICP) e a "Association des Consommateurs de l'Ile Maurice"(ACIM). Nos últimos anos, estas associações os consumidores sobre problemas da segurança de alimentos através da mídia de massa (Vytelingum et al. 2000). Eles também lidam com reclamações de consumidores relacionadas a alimentos, participam de seminários relativos a assuntos alimentícios no nível nacional, regional e internacional e estão envolvidos na redação das normas da MSB

As Associações Comerciais, como a Associação de Fabricantes Mauricianos, a Association des Producteurs et Exportateurs des Produits Horticoles de L'Ile Maurice (APEXHOM), a Câmara de Comércio e Indústria de Maurício (MCCI) e a Câmara da Agricultura de Maurício (MCA) concedem um poder de barganha a seus membros e prestam serviços, incluindo assistência jurídica e suporte técnico. A contribuição destes órgãos para a profissionalização do setor de alimentos está sendo reconhecida. Alguns deles também participam indiretamente do controle de alimentos ao comparecer a comitês, enviar seus comentários e organizar programas de treinamento sobre questões alimentícias.

\section{Instituições de pesquisa}

A Universidade de Maurício empreende a pesquisa no campo de controle de alimentos, mas uma grande parte de seu trabalho não é publicada. O Conselho de Pesquisa Agrícola e de Alimentos (FARC) financia a 
pesquisa sobre os aspectos técnicos da produção. Ele fornece consultoria política ao Ministério da Agro-indústria e de Pesca e coordena e presta suporte à pesquisa e ao desenvolvimento no setor agrícola e de alimentos (FARC 2008).

\section{Estudos sobre o Sistema de Controle de Ali- mentos Mauriciano}

Estudos sobre o MFCS foram realizados de 1985 a 2002 (Dhamija 1985; Gajadhur 1998; Vytelingum 2000; Peersia 2001; OMS 2002). Dhamija (1985) forneceu uma imagem bastante negativa do FCS em 1985. Ele ressaltou que muitas portarias e regulamentos tinham sido emitidos parte por parte, não cobriam todos os aspectos relacionados a alimentos e estavam tendo seu cumprimento executado por diversas agências, além disso a mão-de-obra envolvida no processo era inadequada. Uma falha deste estudo era que a abordagem metodológica usada era unilateral, já que apenas as opiniões das partes interessadas do lado regulador do FCS eram consideradas.

Gajadhur (1998) posteriormente tentou identificar os componentes do MFCS. Este estudo mapeou, primeiro, o controle de alimentos antes da introdução da Lei de Alimentos em 1998. Ele mostrou que existiam diversas leis que estavam sendo usadas no campo do controle de alimentos ${ }^{4}$, sendo a principal a Lei de Alimentos e Medicamentos de 1940. O Ministério da Saúde e Qualidade de Vida (MOH) foi a principal agência coercitiva para a Lei de Alimentos e Medicamentos, embora as autoridades locais tivessem um papel a desempenhar na emissão das licenças para as instalações alimentícias sob a Lei de Classificação do Comércio e das Indústrias de 1954. Como o estudo anterior, este estudo também confiou nas informações coletadas das autoridades e, portanto, mostra apenas uma parte da situação do FCS.

O MFCS posteriormente foi descrito on-line (Vytelingum 2000), com relação ao Projeto da Internet da Lei de Alimentos (FLIP). É interessante observar que esta tentativa de descrever os componentes do sistema de controle de alimentos ocorreu quando a Lei de Alimentos de 1998 e seus regulamentos tinham acabado de entrar em vigor, durante uma fase de transição quando os principais agentes de controle de alimentos ainda estavam se ajustando à nova lei. A descrição enfocou a Lei de Alimentos e seus regulamentos, sua administração e cumprimento, bem como a função de outros corpos de sustentação.

Peersia (2001), então, conduziu uma análise de cenário (Strenghts Weaknesses Opportunities and Threats - SWOT) do MFCS. Além da Lei de Alimentos de 1998, ela considerou outras leis relacionadas a alimentos, como a Lei de Comércio Justo de 1980 e os órgãos envolvidos na administração e no cumprimento das diferentes leis, assim como também o controle voluntário. De acordo com Peersia (2001), existiram muitos problemas no nível de gerenciamento e execução da lei de alimentos apesar desta ser adequada. O estudo mostrou que Maurício tinha conseguido melhorar sua legislação de alimentos.

A OMS (2002) realizou avaliação do FCS baseado em um método de definição de perfil estabelecido (OMS 1989) usando questionário auto-administrados. Este perfil da OMS representa uma visão unilateral do MFCS que é a do MOH.

Para estudar a evolução de um FCS recomenda-se o uso de uma metodologia onde as opiniões dos stakeholders que compõem o FCS são levadas em consideração. FAO/OMS forneceu as diretrizes sobre como estabelecer um FCS. FAO (2006) desenvolve e complementa as diretrizes anteriores da FAO/OMS. Eles recomendam o uso de uma nova abordagem que estabelece a necessidade de auditorias no nível das indústrias de alimentos (OMC 2008). Assim, as diretrizes enfocam o governo e as autoridades de controle de alimentos e permitem uma auto-avaliação. Nós desenvolvemos e construímos ainda sobre estas duas diretrizes e aplicamos uma análise das partes interessadas para avaliar o FCS em Maurício. Até onde sabemos, nenhuma análise foi realizada para medir a percepção das diferentes partes interessadas sobre o MFCS e seus componentes. Esta metodologia é mais sistemática e fornece uma melhoria sobre os estudos anteriores.

\section{Metodologia}

O objetivo principal do trabalho é reformulado de acordo com as seguintes perguntas da pesquisa:

- Qual é a situação do sistema de controle de alimentos local?

- Uma análise dos stakeholders pode ser usada como ferramenta para avaliar o sistema local de controle de alimentos?

É considerada a hipótese de que o MFCS é satisfatório. Considerando que os stakeholders são especialistas em seus respectivos campos e espera-se que eles forneçam um objetivo e uma avaliação confiável dos riscos dos alimentos e de como estes podem ser tratados pelos sistemas de alimentos, a hipótese de que uma análise de partes interessadas pode ser usada para avaliar sistematicamente os MFCS é levada em consideração.

\section{Análise dos stakeholders}

Os stakeholders são indivíduos, grupos ou instituições que têm um interesse em, ou influência sobre a segurança e a qualidade dos alimentos. Eles incluem:

- aqueles que exercem um papel direto e de liderança na administração do controle de alimentos (ministérios governamentais, departamentos, agências);

- aqueles que exercem um papel secundário ou de apoio, tais como os grupos que fornecem informações usadas por aqueles responsáveis pelo controle de alimentos;

- aqueles que são afetados pela administração de controle de alimentos (organizações de consumidores, indústria de alimentos e grupos comerciais, instituições acadêmicas e científicas). 
A análise de stakeholders é uma técnica sugerida por FAO (2006) para identificar e avaliar a importância dos grupos de pessoas e instituições com influência na administração de controle de alimentos. É o processo de juntar sistematicamente e analisar as informações qualitativas para determinar os interesses que devem ser levados em conta ao desenvolver e/ou implementar uma política ou programa (Schmeer 1999) e facilitar os processos de reforma política e institucional (Banco Mundial 2001). Até onde sabemos, esta técnica ainda não foi aplicada para avaliar um NFCS.

\section{Técnicas e ferramentas de coleta de dados}

A coleta de dados se baseou em entrevistas presenciais individuais com aplicação do questionário dos principais participantes na área específica da política do FCS. Isto gerou informações úteis e precisas sobre o MFCS.

Com base na revisão da literatura, foi possível traçar uma lista das questões associadas com o NFCS. Conforme proposto por Jukes (2000), o questionário cobriu todos os quatro componentes principais de um FCS: legislação de alimentos, administração, execução e a função dos órgãos de suporte. O fluxo das informações, a participação nas atividades das organizações nacionais e internacionais e a transparência do FCS também foram avaliados. Uma escala de classificação (valores de 1 a 7: 1-2: muito ruim; 3-5 adequado; 6-7: muito bom) foi usada para perguntar aos respondentes sobre sua opinião em termos de um conjunto de categorias de respostas ordenadas que reflitam a intensidade da opinião particular. Uma escala de sete pontos, ao invés da normal de cinco pontos (Oppenheim 1992) foi usada para reduzir a tendência para agrupamento de respostas na extremidade superior da escala (Henson \& Traill 1999). Além disso, os entrevistados também foram convidados a identificar restrições encontradas e possíveis soluções para os problemas que eles estavam enfrentando atualmente para cumprir com suas responsabilidades. Os envolvidos na análise incluíram representantes de:

- Ministério da Saúde e Qualidade de Vida

- Ministério da Agro-indústria e Pesca

- Agência de Normas de Maurício

- Laboratórios Públicos

- Organizações de Defesa do Consumidor

- Organizações Comerciais

- Instituições de Pesquisa

- Órgãos Profissionais

- Representantes da indústria de alimentos (fabricação, distribuição, importação e exportação)

Em determinados casos, uma organização que representasse os interesses de um grupo entrevistada, ao invés de reunir todos os participantes. Um número total de 22 respondentes, 11 do lado regulador e 11 do lado não-regulador, foi entrevistado.

\section{Análise de Dados}

A média, mediana e faixa de respostas fornecidas pelas partes interessadas foram computadas usando SPSS 14.0. Foi realizada uma tabulação cruzada para medir a associação entre o parâmetro: "categoria" (quer o respondente fosse um órgão regulador ou não-regulador) e afiliação com diversos outros parâmetros sob o estudo a fim de interpretar onde a diferença estava. Como o número de respondentes era inferior a 30, testes não-paramétricos foram realizados usando o Teste $U$ de Mann-Whitney, que é um dos mais criteriosos testes nãoparamétricos para comparar dois grupos de stakeholders (Moore \& McCabe 2003).

\section{Análise da Confiabilidade e Construção de Índice}

Henson e Traill (1999) observaram que alguns aspectos dos alimentos podem ser considerados como construções psicológicas que podem ser medidas ao considerar o nível de concordância com uma série de declarações, cujas pontuações podem ser usadas para desenvolver um índice. Esta técnica de escalonamento somado de múltiplos itens foi aplicada na avaliação do sistema de controle de alimentos com base nas respostas dadas pelas partes interessadas. Esta técnica foi uma forma de aumentar a confiabilidade das escalas Likert. Isto ocorre porque a soma (ou média) de vários ítens deveria ser mais precisa do que a resposta a uma única pergunta, desde que, é claro, todos os ítens usados sejam genuinamente provocados pela construção em questão (Oppenheim 1992).

Há uma variedade de maneiras nas quais a confiabilidade pode ser avaliada (Frankfort-Nachmias \& Nachmias 1996), sendo o alfa de Cronbach o mais comumente usado. O limite superior para alfa aproxima-se de 1 , com valores acima de 0.7 geralmente aceitos como demonstrando que a escala está internamente consistente ou confiável (Frankfort-Nachmias \& Nachmias 1996). Cada construção foi examinada usando os procedimentos detalhados acima por SPSS 14.0 de forma a eliminar os ítens supérfluos. Os ítens com um valor alfa Cronbach baixo não foram incluídos no cálculo do índice de controle de alimentos global.

Os valores numéricos ( 1 a 7 ) foram designados para as respostas das perguntas. Estes valores foram somados para obter as pontuações totais para cada respondente. As pontuações foram, então, interpretadas como indicadores das atitudes dos respondentes nos FCS. Quando a pontuação total de todas as partes interessadas para todas as interpretações forem computadas, a pontuação geral para o sistema de controle de alimentos pode ser obtida. Este número pode ser expresso como um percentual da pontuação para um sistema de controle de alimentos ideal, que é a base para a comparação. A pontuação total para um sistema de controle de alimentos ideal pode ser calculada multiplicando o número de interpretações e o número de partes interessadas pela pontuação máxima, ou seja, $28 * 22 * 7$. O índice assim desenvolvido pode ser usado como um indicador do estado do sistema de controle de alimentos. 


\section{Resultados}

\section{Afiliação dos stakeholders}

Cinco, dois, quatro e onze partes interessadas eram, respectivamente, do setor privado, ONGs e sociedade civil, organizações paraestatais e órgãos governamentais.

\section{Situação do controle de alimentos e segu- rança dos alimentos}

Os respondentes foram questionados sobre sua percepção quanto à situação atual do controle e segurança dos alimentos em Maurício. Com base nas classificações médias e medianas, as partes interessadas avaliaram a situação do controle de alimentos e da segurança dos alimentos como adequadas, com uma pontuação média de 4.05 e 3.73 e uma contagem mediana de 4.00 e 4.00 , respectivamente.
Havia diferenças entre as médias e as medianas dos dois grupos de respondentes (órgãos reguladores e nãoreguladores) em relação à avaliação sobre o estado do controle e segurança de alimentos em Maurício (Tabela 1). Mas, com base no teste U de Mann Whitney, havia uma diferença estatisticamente significativa no nível de $5 \%$ entre a resposta dos órgãos reguladores e dos nãoreguladores para apenas a situação da segurança dos alimentos em Maurício.

\section{Situação dos componentes do sistema de controle de alimentos}

Os stakeholders classificaram os diferentes componentes do FCS como adequados (Tabela 2). A “Execução da Lei de Alimentos" obteve a pontuação mais baixa, enquanto os "instrumentos jurídicos" obtiveram a pontuação mais alta.

Tabela 1 - Situação do controle de alimentos e segurança de alimentos

\begin{tabular}{ccc}
\hline \multirow{2}{*}{ Parâmetro } & \multicolumn{2}{c}{ Resposta Média (Mediana) fornecida por } \\
& Órgãos Reguladores $(\mathbf{n}=1 \mathbf{1})$ & Órgãos Não-Reguladores $(\mathbf{n}=11)$ \\
\hline Situação do controle de alimentos & $4.27(4.00)$ & $3.18(3.00)$ \\
Segurança de alimentos & $4.64(5.00)^{*}$ & $3.45(4.00)^{*}$ \\
\hline
\end{tabular}

Nota: (1-2: Muito ruim, 3-5: Adequado; 6-7: Muito Bom)

*: Pontuações estatisticamente diferentes no nível de 5\%.

Tabela 2 - Avaliação dos componentes dos sistemas de controle de alimentos

\begin{tabular}{cccc}
\hline & Stakeholders & $\begin{array}{c}\text { Órgãos Reguladores } \\
(\mathrm{n}=11)\end{array}$ & $\begin{array}{c}\text { Órgãos Não-Regula- } \\
\text { dores }(\mathrm{n}=11)\end{array}$ \\
\hline Componente & Média (mediana) & $4.64(5.00)$ \\
\hline Instrumentos Jurídicos & $5.00(5.00)$ & $5.36(6.00)$ & $2.91^{*}(3.00)$ \\
Administração da lei de alimentos & $3.36(3.00)$ & $3.82^{*}(4.00)$ & $2.55^{*}(2.00)$ \\
$\quad \begin{array}{c}\text { Execução da lei de alimentos } \\
\text { Órgãos de Suporte }\end{array}$ & $3.09(3.00)$ & $3.64^{*}(3.00)$ & $4.00(4.00) \#$ \\
$\quad$ Serviços analíticos & & & $3.27(3.00) \#$ \\
Organizações de Defesa do Consumidor & $3.18(4.00)$ & $3.36(4.00) \#$ & $3.55(3.00) \#$ \\
Instituições de Pesquisa & $3.55(3.00)$ & $3.55(3.00) \#$ & $4.18(4.00) \#$ \\
Organizações profissionais (incluindo \\
associações comerciais)
\end{tabular}

Nota: (1-2: Muito ruim, 3-5: Adequado; 6-7: Muito Bom)

\#: mesma mediana

*: Pontuações estatisticamente diferentes no nível de $5 \%$ baseadas no teste U de Mann-Whitney

A resposta média entre os dois grupos de stakeholders foi diferente (Tabela 3). De acordo com órgãos não-reguladores, o componente com a menor pontuação foi "execução" (mediana $=2.00$, ou seja, muito deficiente).

Baseado na contagem mediana, as partes interessadas de órgãos reguladores consideravam o "cumpri- mento" adequado, enquanto as partes interessadas das organizações não-reguladoras consideravam-no muito deficiente. O Teste U de Mann-Whitney mostrou que a diferença entre a resposta dos órgãos reguladores e a dos órgãos não-regulador era estatisticamente significativa no nível de 5\% para os componentes da "administração" e de "cumprimento" apenas. 
Conformidade com a Lei de Alimentos de 1998 e adoção das Boas Práticas de Fabricação e HACCP

Com respeito à conformidade da indústria de alimentos com a Lei de Alimentos de 1998 e seus regulamentos, a maioria dos respondentes classificou a conformidade de grandes fabricantes de alimentos, exportadores e grande indústria hoteleira e de catering como "muito boa" (Tabela 3). Algumas partes interessadas acreditavam que os grandes importadores de alimentos poderiam estar aceitando alimentos a um preço abaixo do mercado, explicando, provavelmente, a menor pontuação concedida a eles em comparação com os grandes fabricantes de alimentos, grandes indústrias hoteleiras e de catering e exportadores de alimentos.

Tabela 3 - Conformidade com a Lei de Alimentos de 1998 e Regulamentos de Alimentos de 1999

\begin{tabular}{|c|c|c|c|}
\hline Tipo de indústria: & Média & Faixa & Mediana \\
\hline Grandes Fabricantes de Alimentos & 5.50 & $3-7$ & 6.00 \\
\hline Importadores de Alimentos grandes & 4.86 & $3-6$ & 5.00 \\
\hline Exportadores & 5.59 & $4-7$ & 6.00 \\
\hline Fabricantes de Alimentos de Pequena e Média Escala & 3.77 & $2-6$ & 4.00 \\
\hline Indústria Hoteleira e de Catering (Grande) & 5.64 & $4-7$ & 6.00 \\
\hline Indústria Hoteleira e de Catering (Pequenas e Médias) & 3.91 & $2-6$ & 4.00 \\
\hline Setor Informal de Alimentos & 1.82 & $1-3$ & 2.00 \\
\hline
\end{tabular}

(1-2: Muito ruim; 3-5 Adequado; 6-7: Muito bom)

Houve convergência nas percepções das partes interessadas quanto à conformidade muito deficiente do setor informal de alimentos com a legislação de alimentos, já que dez de onze órgãos reguladores e nove de 11 órgãos reguladores consideravam que o setor informal de alimentos cumpria de forma muito deficiente a legislação local sobre alimentos.

Com relação à adoção de Boas Práticas de Fabricação ou Ponto de Controle Crítico de Análise de Risco (HAC$\mathrm{CP}$ ), os grandes fabricantes de alimentos, exportadores e grande indústria hoteleira e de catering foram considerados como tendo um nível muito bom de adoção, enquanto as pequenas e médias indústrias alimentícias e operadores de catering tinham um nível adequado de adoção.

\section{Participação no sistema de controle de alimentos e envolvimento na configuração padrão}

Os stakeholders avaliaram o seu grau de participação no sistema de controle de alimentos como adequado $($ Média $=4.77 ;$ Mediana $=5.00)$. Das vinte e duas partes interessadas, 12 partes interessadas tinham participado dos comitês pertencentes à segurança de alimentos realizados pelo $\mathrm{MOH}$.

As partes interessadas estavam satisfeitas (Média = 3.68; pontuação mediana $=4.00$ ) com seu envolvimento na configuração padrão no nível da MSB em Maurício. As respostas variaram de 1 a 6 , com sete das vinte e duas partes interessadas se considerando como tendo um envolvimento muito pobre na configuração padrão.

\section{Mecanismo institucional dentro do país para consulta e desenvolvimento de políticas sobre os regulamentos nacionais sobre alimentos}

Em média, as partes interessadas estavam satisfeitas $($ Média $=3.73$; pontuação mediana $=3.50)$ com o mecanismo institucional para consulta e desenvolvimento de políticas sobre regulamentos nacionais sobre alimentos com dez das vinte e duas partes interessadas considerando-o adequado.

A Tabela 4 apresenta a diferença nas pontuações médias e medianas das partes interessadas com diferentes antecedentes. Baseados em pontuações medianas, os órgãos reguladores consideravam que o mecanismo institucional para consulta e desenvolvimento de política era adequado (pontuação mediana $=5.00$ ) enquanto os órgãos não-reguladores o consideravam muito deficiente (pontuação mediana $=2.0$ ). Havia uma diferença estatisticamente significativa nas pontuações dos dois grupos no nível de 5\% com base no Teste U de Mann Whitney.

\section{Oportunidades para tornar as opiniões conhe- cidas dentro do Codex através do Governo}

Oito dos vinte e dois respondentes consideravam que as oportunidades para tornar as opiniões conhecidas dentro do Codex através do governo eram muito insuficientes. 
Em média, os respondentes consideravam que eram adequadas (Média $=3.45$; Mediana $=3.0$ ). Os respondentes que submeteram suas opiniões determinaram que eles assim o fizeram através do Ponto de Contato Codex.

Baseados nas pontuações medianas, os órgãos reguladores consideraram que as oportunidades para tornar as opiniões conhecidas para o Codex através do
Governo eram adequadas (pontuação mediana $=5.00$ ) mas os órgãos não-reguladores as consideraram muito insuficientes (pontuação mediana $=2.00$ ). Havia uma relação estatisticamente significativa no nível de 5\% entre a categoria das partes interessadas e sua satisfação com o grau de satisfação com a oportunidade das partes interessadas para tornar suas opiniões conhecidas.

\section{Tabela 4 - Satisfação com o mecanismo institucional para consulta e desenvolvimento de políticas}

\begin{tabular}{cccc}
\hline \multicolumn{1}{c}{ Parâmetro } & \multicolumn{1}{c}{ Média (mediana) } \\
\hline $\begin{array}{c}\text { Satisfação com o mecanismo institucio- } \\
\text { nal para consulta e desenvolvimento de } \\
\text { políticas }\end{array}$ & $3.73(3.50)$ & $4.55^{*}(5.00)$ & $2.91^{*}(2.00)$ \\
$\begin{array}{c}\text { Oportunidades para tornar as opiniões } \\
\text { conhecidas dentro do CÓDICE através do } \\
\text { Governo }\end{array}$ & $3.45(3.00)$ & $4.55^{*}(4.00)$ & $2.36^{*}(2.00)$ \\
\hline
\end{tabular}

Nota: (1-2: Muito insuficiente, 3-5: Adequado, 6-7: Muito Bom)

*: Pontuações estatisticamente diferentes no nível de 5\% baseado no teste de U de Mann-Whitney

\section{Fluxo de informações}

A resposta média para o fluxo de informações dos órgãos reguladores para o público sobre o sistema de controle de alimentos (indicativo do grau de transparência) foi de 2.91 (mediana $=3.0$ ), com sete respondentes fornecendo 1 e 2 pontos. Nenhuma das partes interessadas entrevistadas considerou o fluxo de informações em Maurício sobre o FCS como "muito bom". A pontuação média dos órgãos não-reguladores (2.64) era menor em comparação com os stakeholders dos órgãos reguladores (3.18).

\section{Análise de confiabilidade e índice para o sistema de controle de alimentos}

Um índice de controle de alimentos (FCI) para medir a condição dos MFCS foi desenvolvido a partir das respostas das partes interessadas a todas as perguntas. Os ítens selecionados após realizar o teste de confiabilidade alfa de Cronbach incluíram o comportamento das partes interessadas para com o seguinte:

- Situação atual do controle de alimentos

- Qualidade e segurança dos alimentos

- Instrumentos jurídicos projetados para o controle de alimentos

- Administração da lei sobre alimentos

- Execução da lei sobre alimentos

- Papel dos órgãos de suporte (órgãos analíticos, organizações do consumidor, instituições de pesquisa e órgãos profissionais)
- Fluxo de informações

- Nível de participação

- Envolvimento no estabelecimento da política Codex

- Satisfação com o mecanismo institucional para consulta e desenvolvimento de políticas

- Conformidade com as leis e regulamentos pertinentes (não para todas as categorias da indústria de alimentos)

- Auto regulação na indústria de alimentos (não para todas as categorias da indústria de alimentos)

Baseado nas estatísticas de confiabilidade, era razoável desenvolver o FCI baseado em todas as 28 interpretações, já que todas as escalas eram internamente compatíveis e confiáveis como o coeficiente a global eram satisfatoriamente maiores do que 0.7. O FCI se baseou no princípio da agregação (Frankfort-Nachmias \& Frankfort 1996) e foi calculado:

$\mathrm{FCl}=\frac{\text { Total score for MFCS }{ }^{*} 100}{\text { Number of stakeholders }{ }^{*} \text { max imum score }{ }^{*} \text { number of constructs }}$

Tradução da fórmula acima:

FCI = Pontuação total para MFCS * 100 / Quantidade de partes interessadas * pontuação máxima * número de interpretações

O FCI para Maurício era de 58.5\%. Assim, o MFCS pode ser classificado como FCS Classe $\mathrm{C}^{5}$. 


\section{Discussão}

\section{Situação geral do sistema de controle de alimentos de Maurício}

Baseado no FCI global resultante deste estudo, há evidBaseado no FCI global resultante deste estudo, há evidências de que Maurício tem um FCS satisfatório (classe C). De fato, a situação do controle e da segurança de alimentos no país foram consideradas adequadas pelos stakeholders locais. Porém, havia uma diferença estatisticamente significativa nas opiniões expressas pelas partes interessadas de órgãos reguladores e aquelas dos órgãos não-reguladores com respeito à situação da segurança dos alimentos. Esta diferença na percepção pode ser atribuída ao fato de que há um dualismo no suprimento de alimentos domésticos. Por um lado, existem operadores alimentícios em pequena e média escala e o setor informal de alimentos. A maioria dos operadores no setor informal de alimentos é privada de conhecimentos básicos sobre higiene ou não cumpre com os regulamentos da segurança dos alimentos. Por outro lado, há operadores de grande escala que possuem um alto grau de conformidade com a legislação local de alimentos e adotaram práticas de auto regulação, conforme realçado pela análise das partes interessadas. Portanto, a análise das partes interessadas expôs uma área problema.

Outro ponto a considerar é que as partes interessadas das organizações não-reguladoras distinguiram entre a segurança dos alimentos disponíveis no setor alimentício informal local e os alimentos importados. O último foi considerado geralmente seguro e de alta qualidade, enquanto o primeiro apresentava segurança deficiente já que as cadeias frias não estavam sistematicamente presentes. As partes interessadas também indicaram que havia um mecanismo para monitorar as importações, mas ele foi limitado por uma escassez de pessoal treinado. Este resultado concorre com a OMS (2002). Os dados sobre as detenções e rejeições sobre as consignações de alimentos não são abrangentes, ao contrário dos países desenvolvidos como os Estados Unidos, onde tais dados são compilados e disponibilizados publicamente ${ }^{6}$.

\section{Legislação de alimentos}

A classificação média para o componente "legislação de alimentos" era "adequada" (5.00). Na realidade a legislação de alimentos era o componente com a pontuação média mais alta e sem qualquer divergência entre as partes interessadas a partir de cenários reguladores e não-reguladores. Isto pode ser explicado pelo fato que a lei é moderna e contém a maioria dos requisitos essenciais de uma lei de alimentos ideal. Já que o sistema legislativo não sustentou o controle de alimentos por um longo período em Maurício, a nova lei foi aclamada. A Lei de Alimentos de 1998 foi adaptada da lei de alimentos do Reino Unido, mas diferentemente do último ela não evoluiu constantemente, mas, ao invés disso, foi drasticamente modernizada em 1998 com um texto total e consolidado para os regulamentos.
Também foi indicado que a lei de alimentos em si era boa, mas o único ponto de limitação se relacionava à forma como ela tinha sido redigida e apresentada para a indústria.

\section{Administração e execução}

Havia uma divergência estatisticamente significativa na percepção da "administração" e "cumprimento" entre as partes interessadas "reguladoras" e "não-reguladoras". Os resultados indicam que a administração e o cumprimento são duas áreas problema. Isto pode ser atribuído à ausência de uma estratégia nacional para a segurança dos alimentos. Assim, a administração e a execução sofrem da falta de coordenação e duplicação de trabalho, levando a um desperdício de recursos e lacunas na cobertura. Entrevistas detalhadas com partes interessadas revelaram que alguns destes problemas já existiram antes da introdução da Lei de Alimentos de 1998, enquanto outros surgiram ou foram exacerbados com a nova lei de alimentos. Estes resultados mostram ainda que os participantes proviam de dois cenários distintos. Cada grupo de partes interessadas avaliou favoravelmente os parâmetros relacionados a eles. Por exemplo, as partes interessadas das autoridades reguladoras deram contagens mais altas para a execução (3.00) em comparação com as partes interessadas dos órgãos não-reguladores (2.00).

\section{Órgãos de suporte}

O MFCS exige a ajuda de certos órgãos de suporte para ser totalmente eficaz. Não existe, porém, qualquer estrutura que defina os papéis e responsabilidades dos diferentes órgãos de suporte com relação ao FCS. Todas as categorias de órgãos de suporte foram pontuadas como sendo geralmente "adequadas". Porém, a entrevista revelou uma série de inadequações no nível dos órgãos de suporte. Isto demonstra a relevância do uso de metodologiascomo a análise de stakeholders e entrevistas detalhadas, como neste estudo.

\section{Órgãos analíticos}

Vale a pena mencionar que os resultados das análises de partes interessadas mostram que não houve grandes melhorias nas instalações de laboratório existentes localmente, em comparação com os resultados de estudos anteriores (Peersia 2001; Saib 2002; SADC 2002). Algumas partes interessadas foram críticas sobre as instalações de laboratório existentes em Maurício, considerando-as equipadas de forma deficiente, não fazendo uso dos modernos métodos analíticos ou procedimentos adequados para garantia de qualidade. É importante observar que embora uma série de laboratórios governamentais e paraestatais estejam envolvidos nos testes de alimentos localmente, somente os laboratórios $\mathrm{MOH}$ são reconhecidos de acordo com a Lei de Alimentos de 1998. As partes interessadas também foram convencidas de que a operação plena do Laboratório de Tecnologia de Alimentos, sendo estabelecida sob MOA, iria melhorar a situação. Porém, isto teria que ser reconhecido formalmente pelo $\mathrm{MOH}$ também. 


\section{Organizações de comércio, órgãos profissionais e instituições de pesquisa}

Os órgãos profissionais geralmente eram considerados adequados. Este estudo mostrou que havia uma falta de órgãos profissionais como a International Food Science and Technology, que presta suporte técnico para a indústria de alimentos, desenvolvedores e fiscalizadores de políticas. $\mathrm{Na}$ realidade, as associações de comércio assumem uma grande função localmente para melhorar o FCS. Por exemplo, a Câmara da Agricultura de Maurício categorizou os vários setores de alimentos e está trabalhando para fomentar o crescimento de pequenos e médios empreendimentos através do conceito de agrupamento. Porém, as partes interessadas consideraram que o foco destas associações estava no aumento do volume da produção de alimentos e não necessariamente no aumento da segurança de alimentos.

Entre os órgãos de suporte, as instituições de pesquisa receberam a pontuação mais baixa. As partes interessadas eram da opinião de que as instituições de pesquisa não eram bem organizadas. Não havia qualquer estratégia nacional para a pesquisa de alimentos no contexto local e não havia ali qualquer mecanismo para ajudar as instituições a contribuírem para o controle de alimentos. Embora a segurança de alimentos tenha sido identificada por uma instituição como uma área prioritária, foi impossível pesquisá-la por causa da falta de fundos, pessoal e infra-estrutura. No momento, parecia que as instituições de pesquisa somente poderiam participar do controle de alimentos através de sua entrada em comitês organizados pelos órgãos reguladores, tais como o National Codex Committee (MOA) e pelo Órgão Nacional de Normas, ou seja, o Comitê de Alimentos e Agrícola (MSB). Porém, deve ser mencionado que a Universidade de Maurício está ativamente envolvida em aprofundar o conhecimento na ciência de alimentos através de suas atividades de treinamento e pesquisa.

\section{Organizações de Consumidor}

A média de resposta dos órgãos reguladores e nãoreguladores foi a mesma para suas avaliações sobre as organizações de consumidores, indicando uma convergência de percepções. O envolvimento destas organizações atualmente estava muito limitado devido à falta de pessoal qualificado e por motivos financeiros. Além disso, eles não tinham o suporte científico adequado.

\section{Implementação da Lei de Alimentos de 1998 e regulamentos pela indústria de alimentos local}

Houve uma grande concordância entre as partes interessadas dos órgãos reguladores e não-reguladores sobre a situação do cumprimento de todas as categorias de negócios de alimentos locais com a legislação local de alimentos. Todos concordaram sobre o cumprimento muito deficiente do setor informal de alimentos com a legislação local. As opiniões dos respondentes sobre a situação da auto-regulação entre todas as categorias da indústria de alimentos também convergiram. A autoregulação é usada pelos fabricantes locais como uma ferramenta competitiva e para melhorar a segurança do produto. O fato de que Maurício é um destino turístico obriga a grande indústria hoteleira e de catering a adotar tais sistemas de administração da segurança de alimentos como uma garantia para seus clientes. Os exportadores são obrigados a adotar sistemas, tais como HACCP, porque é obrigatório em seu mercado de exportação.

Os grandes fabricantes de alimentos, grandes exportadores de alimentos e a grande indústria hoteleira e de catering em Maurício estabeleceram práticas voluntárias muito boas, isto é, a auto-regulação. Nossos resultados, portanto, estão alinhados com os relatórios anteriores (Chung 2000; Baumy 2001; Peersia 2001; Dooky 2001) sobre a conformidade da indústria de alimentos local com a legislação de alimentos e o estabelecimento de sistemas voluntários de administração da segurança de alimentos nas grandes indústrias de alimentos e no grande setor hoteleiro. Dooky (2001), em um estudo que compreendia a implementação dos Regulamentos de Alimentos em grandes hotéis, concluiu que a maioria dos hotéis pesquisados eram capazes de satisfazer os novos requisitos de acordo com os regulamentos já que eles já tinham uma abordagem integrada implementada para administração da segurança de alimentos.

Havia uma visão unânime sobre a conformidade deficiente do setor informal de alimentos com a legislação. Os resultados deste estudo confirmam as conclusões de estudos anteriores com relação ao setor de alimentos informal (Jugessur et al. 2000; Joomun 2001; Duffaydar 2001; Rumjaun 2001). A falta de controle sobre o setor informal em um país também pode ser um fator limitador do funcionamento adequado de um FCS (Igbedioh \& Akinyele 1992). A conveniência de e o acesso aos alimentos disponíveis pelo canal informal poderia explicar por que os mauricianos adquirem alimentos do setor informal.

\section{Participação no sistema de controle de alimentos, fluxo de informações e interação com as organizações que desenvolvem as normas internacionais}

As partes interessadas geralmente estavam satisfeitas com a sua participação no FCS e seu papel no estabelecimento de normas. Isto indicou que cada stakeholder estava ciente de sua função e responsabilidade no FCS e lutava por encontrar aquelas dentro dos recursos disponíveis. As partes interessadas deram uma pontuação baixa para os critérios de "fluxo de informações”, mostrando que a transparência ainda é um problema em Maurício. Embora informações suficientes possam estar disponíveis, a disseminação não é boa. Em Maurício, os recursos são tão limitados que eles raramente são usados para promover o fluxo de informações. Foi identificado que a falta do livre fluxo de informações entre as agências envolvidas no controle de alimentos no Kuwait era uma restrição importante (Alomirah et al. 2004). Normalmente em países em desenvolvimento, os canais formais de comunicação 
entre as organizações de controle de alimentos e os consumidores geralmente não existem ou existem apenas através de reclamações do consumidor. Esta fato pode criar apreensões desnecessárias. Ao invés disso, diversos tipos de informações poderiam ser compartilhados entre as partes interessadas e usadas para educar o público, por exemplo, informações relativas à taxa de conformidade dos negócios alimentícios. Isto poderia ajudar a impulsionar a confiança dos stakeholders no FCS.

Havia também uma diferença estatisticamente significativa na resposta das partes interessadas dos órgãos reguladores e não-reguladores com relação ao mecanismo institucional para consulta e desenvolvimento de políticas sobre padrões e regulamentos nacionais de alimentos. Isto poderia ser atribuído ao fato de que as partes interessadas do lado não-governamental acreditavam que não havia tido consulta o suficiente antes da introdução da nova legislação de alimentos. Além disso, havia uma diferença estatisticamente significativa na resposta das partes interessadas dos órgãos reguladores e não-reguladores com relação a suas oportunidades para tornar as opiniões conhecidas para o Codex através do governo. Isto provavelmente se devia ao fato de que havia muito pouca satisfação com a sua capacidade para tornar as opiniões conhecidas e que nem todas as partes interessadas estavam envolvidas no estabelecimento de políticas do Codex.

\section{Análise dos stakeholders}

Este estudo demonstrou o uso da análise dos stakeholders como uma ferramenta potencial para a avaliação do FCS. Cada grupo de partes interessadas avaliou favoravelmente os parâmetros relacionados a elas. Por exemplo, as partes interessadas das autoridades reguladoras concederam pontuações maiores para a legislação de alimentos em comparação com as partes interessadas de órgãos não-reguladores, mas esta divergência nas percepções eram estatisticamente significativas no caso da avaliação do estado da segurança de alimentos, administração e componentes de execução, consulta e oportunidades para tornar as opiniões conhecidas dentro do Codex através do Governo. Esta divergência de opiniões pode ser um indicativo de uma área problema. Outra razão que poderia responder pela diferença de opinião era a falta do fluxo de informações entre as partes interessadas dos órgãos reguladores e não-reguladores.

É, importante, porém, incluir as partes interessadas de ambos os setores ao preparar qualquer estratégia de controle de alimentos ou legislação de forma que as suas visões sejam incorporadas. Ao incluir as partes interessadas de ambos os cenários em uma análise de um FCS, uma opinião mais equilibrada e completa é obtida e isto melhora a qualidade e a confiabilidade da avaliação. Esta filosofia concorre com Houghton (2008), que enfatizou a importância de envolver stakeholders na administração do risco de alimentos. A qualidade e a confiança são refletidas no FCI. A metodologia aplicada aqui pode ser usada em outros países para derivar índices de controle de alimentos e assim fazer comparações internacionais de NFCS. Estas informações podem ser usadas pelos
Ministérios responsáveis pelos doadores internacionais e da Saúde para estabelecer e financiar programas.

\section{Conclusão e recomendações}

A partir da premissa de que as partes interessadas da indústria de alimentos são os peritos e muito provavelmente são os mais capazes para fazer julgamentos sobre o FCS, foi feita uma análise de partes interessadas. Um FCI também foi computado baseado nas respostas das partes interessadas. Foi considerado que não havia qualquer diferença estatisticamente significativa entre as médias de respostas dos órgãos reguladores e não-reguladores, com exceção das seguintes variáveis: classificação da situação da qualidade e segurança de alimentos, administração e execução, consulta e oportunidade para tornar as opiniões conhecidas para o Codex através do Governo. Estes poderiam indicar áreas problema. Baseado no FCI, Maurício tem um FCS satisfatório.

Esta análise de stakeholders apresentou uma condição atualizada do Sistema de Controle de Alimentos de Maurício. É interessante ver como estes resultados concordam com os estudos anteriores, embora seja sabido que as abordagens metodológicas e os prazos de estudos são distintos. De acordo com as partes interessadas, não houve melhoria nos MFCS, exceto pela renovação da legislação de alimentos. A rápida abordagem metodológica permitiu uma avaliação detalhada do sistema. Considerando que nenhum trabalho foi feito antes para desenvolver um FCI e categorizar um FCS, foi autorizada uma pesquisa adicional para cruzar resultados e, também, rastrear a evolução do MFCS com o passar do tempo. Assim, a mesma análise deveria ser executada sistematicamente a cada dois anos. Porém, para que esta metodologia seja eficaz, há uma necessidade de incluir os stakeholders a partir dos domínios reguladores e não-reguladores e fazer uma escolha cuidadosa dos parâmetros a serem usados na formação do índice de controle de alimentos.

\section{Agradecimento}

Ao Dr. A. Ruggoo pela orientação estatística e aos stakeholders do sistema de controle de alimentos local.

\section{Notas}

1. 1 US\$ $\approx 30 \mathrm{MRU}$.

\section{MRU: Rúpias de Maurício}

3. Qualquer artigo ou substância destinado ao consumo humano, inclusive bebidas, água engarrafada, chicletes, artigos e substâncias usadas ou planejadas para uso como ingredientes na composição ou preparação de alimentos, mas que não sejam animais de criações, compostos alimentares, medicamentos ou remédios e produtos hormonais.

4. Estas são as Leis de Saúde Pública (PHA) de 1981 (aditivo), a Lei de Alimentos e Medicamentos de 1940 e os regulamentos das mesmas, por exemplo, os Regulamentos de Alimentos e Drogas (Sal) de 1989 e a Lei de Classificação de Indústrias e Comércio de 1954 e a Lei da Carne de 1974. 
5. Classe A (Muito Boa: 70-100); classe B (Boa): 60-69; classe C (Satisfatória): 50-59; classe D (Deficiente): 4049; classe $\mathrm{E}$ (Muito deficiente) $:<40$.

6. www.fda.gov/ora/import/default.htm

\section{Referências bibliográficas}

ACD-Agricultural Chemistry Division. The Ministry of Agro-Industry and Fisheries, Mauritius, 2008. Disponível em: <http://agriculture.gov.mu/chem.htm>. Acesso em: April 282008.

Alomirah H, Al-Zenki S, Sawaya WN, Hussain A, Omair A. Assessment of the food control system in the state of Kuwait. In: International Food Technologists meeting, July 12-16, 2004, Las Vegas. Proceedings. Dsiponível em: <ift.confex.com/ift/2004/techprogram/session_3169. htm>. Acesso em: April 202008.

Baumy P. A study on food safety management in local large scale food industries. [Dissertation (B.Sc.)]. University of Mauritius: Mauritius, 2001.

Boutriff E, Bessy C. Basic approaches to consumer protection- FAO/WHO model food act control procedures. In: Conference on International Food Trade beyond 2000: Science-based decisions, harmonisation, equivalence and mutual recognition, 11-15 October 1999, Melbourne. Proceedings. Rome: Italy; 1999.

Bruno, A. Review of national import food control systems. In: Food safety and quality control systems-Scientific background and their application for public health, 18-22 November 1996, Warsaw. Proceedings. Joint FAO/ILSIEUROPE workshop, FAO, Rome: Italy; 1996.

Chung MKH. A study on quality management in local large scale food industries. [Dissertation] Mauritius: University of Mauritius; 2000.

DCD-Dairy Chemistry Division. The Ministry of AgroIndustry and Fisheries, Mauritius, 2006. Disponível em: <http://agriculture.gov.mu/dairy.htm > . Acessado em: April 282008.

Dhamija OP. Report on the survey of food control infrastructure in Mauritius. FAO: Rome; 1985.

Dooky BS. A study on the implementation of food regulations 1999 and good manufacturing practices in selected large hotels. [Dissertation] University of Mauritius: Mauritius; 2001.

Duffaydar Z. A study on food safety and quality management in a sub-sector of local small and medium scale food industries. [Dissertation] Mauritius: University of Mauritius; 2001.

DVS- Division of Veterinary Sciences. The Ministry of Agro-Industry and Fisheries, Mauritius, 2008. Disponível em: < http://agriculture.gov.mu/vete.htm>. Acessado em: April 282008.

FARC - Food and Agricultural Research Council. The Ministry of Agro-Industry and Fisheries, Mauritius,
2008. Disponível em: < http://farc.gov.mu/>. Acessado em: April 282008.

FAO. Strengthening national food control systems: guidelines to assess capacity building needs. Food and Agriculture Organisation of the United Nations, Rome. 2006. Disponível em: <www.fao.org/ag/agn/index_en.stm > . Acessado em: January 102008.

FAO/WHO. Guidelines for developing an effective food control system. Rome: FAO. FAO/WHO 1/1; 2003.

Frankfort-Nachmias C, Nachmias D. Index construction and scaling methods. In: Frankfort-Nachmias C, Nachmias D, editors. Research methods in the social sciences. London: St Martin's Press; 1996. p. 455-71.

Gajadhur P. Control of food hygiene by authorities. [dissertation] Mauritius: University of Mauritius; 1998.

Henson S, Traill B. Development of scales to measure perceived performance of the food system and an index of food-related welfare. In: Conference on New Economic Approaches to Consumer Welfare and Nutrition, 14-15 January, 1999, Alexandria. Proceedings of the Conference organised by the Food and Agricultural Marketing Consortium. Washington, D.C.: United States; 1999.

Houghton JR, Rowe G, Frewer LJ, Van Kleef E, Chryssochoidis G, Kehagia O, Korzen-Bohr S, Lassen J, Pfenning U, Strada A. The quality of food risk management in Europe: perspectives and priorities. Food Policy. 2008; 33:13-26.

Igbedioh SO, Akinyele IO. What future for food control in developing countries? Some lessons from Nigeria. Ecol Food and Nutrition. 1992; 27(2):127-132.

Joomun SS. A study of the level of hygiene prevailing among food hawkers in the town of Curepipe. [dissertation] Mauritius; University of Mauritius; 2001.

Jugessur K, Behary K, Bhaugeerothee R. A study of hygienic practices of ready-to-eat food in the city of Port-Louis. [dissertation] Mauritius: University of Mauritius; 2000.

Jukes DJ. Food legislation. Department of Food Science and Technology, FS927. UK: The University of Reading, 2000. Disponível em: <http://www.fst.rdg.ac.uk/foodlaw/fs927/lecture3.htm\# 1 >. Acessado em: August 15 2002.

Kenny M. Elements of an effective export food control system. In: Food safety and quality control systemsscientific background and their application for public health, 18-22 November 1996, Warsaw. Proceedings. Joint FAO/ILSI-EUROPE workshop, , Rome: FAO; 1996. p.13.

MLG. Duties of local authorities. Ministry of Local Government. 2008. Disponível em: < http://localgovernment. gov.mu/local.htm. >. Acessado em: January 282008.

MOH. Duties of health inspectors. Ministry of Health and of the Quality of Life. Port-Louis, Mauritius, 1993. 
MOH. Food Act 1998. Government Gazette, No 64 of 13 June 1998, Mauritius: 1998.

Oppenheim AN. Questionnaire design, interviewing and attitude measurement. London: Cassell; 1992.

Moore DS, Mccabe GP. Introduction to the practice of statistics. 4th Edition. New York: W.H. Freeman and Company; 2003.

Neeliah SA, Goburdhun D. National food control systems: a review. Food Rev Intern. 2007; 23:35-51.

Nguz K. Assessing food safety system in sub-Saharan countries: an overview of key issues. Food Control. 2007;18:131-134.

Peersia KA. Critical evaluation of the food control system in Mauritius. [dissertation] Mauritius: University of Mauritius; 2001.

Ramsamy L. Meeting the requirements of the Food Regulations 1999: a challenge for selected local large scale food industries. [dissertation] Mauritius: University of Mauritius; 2001.

Rees N, Watson D. International standards for food safety. Maryland: Aspen Publication; 2000.

Rumjaun Z. A study on the awareness, constraints and benefits of implementation of the food act among street food vendors. [dissertation] Mauritius: University of Mauritius; 2001.

SADC. Report on harmonisation of SPS/food safety measures in SADC member states: phase I- Inventory of SPS/ food safety measures (Republic of Mauritius). SADC food security and rural development hub, Zimbabwe; 2002.

Saib SB. A study on the enforcement of the food act 1998 and horizontal regulations Food Regulations 1999. [dissertation] Mauritius: University of Mauritius; 2002.
Schmeer K. Stakeholder analysis guidelines. In: Policy toolkit for strengthening health sector reform, Abt Associates, Inc. Bethesda, MD; 1999.

UNDP. Fighting climate change: human solidarity in a divided world. Human Development Report 2007/2008, United Nations Development Programme; 2007.

Vytelingum SA. National food control system: a case study of Mauritius. In: Food Africa Internet Forum, 31 March-11 April; 2003.

Vytelingum SA. The Food Law Internet Project: Mauritius. 2000. Disponível em: http://www.fst.rdg.ac.uk/foodlaw/index.htm. Acessado em: September 242005.

Vytelingum SA, Goburdhun D, Ruggoo A. An assessment of hygiene in animal product processing plants- a case- study of Mauritius. Univ Mauritius Res J. 2000; 6:83-104.

WHO. Evaluation of programmes to ensure food safety. Guiding principles, Geneva: WHO; 1989.

WHO. Food safety country profile for Mauritius. 2002. Disponível em: < http://www.afro.who.int/des/fos/country_profiles/>. Acessado em: 20 April 2008.

World Bank. Stakeholder analysis. 2001. Disponível em: <wwwl.worldbank.org/publicsector/anticorrupt/PoliticalEconomy/PDFVersion.pdf $>$. Acessado em: April 202008.

World Bank. Managing change in a changing world. Mauritius Country Economic Memorandum: World Bank; 2007.

WTO. FAO capacity evaluation tools for food safety, biosecurity and plant protection. Geneva: G/SPS/GEN/831; 2008.

\section{Sobre os autores}

\section{Shalini A. Neeliah}

Shalini Neeliah foi admitida na Divisão de Química de Laticínios como Funcionário Técnico em 2001 e atualmente trabalha como Pesquisa Sênior e Funcionário de Desenvolvimento no Laboratório de Tecnologia de Alimentos do Ministério da Agro-indústria e de Pesca. Depois de sua primeira graduação em Agricultura, ela fez mestrado em Tecnologia de Alimentos na Universidade de Reading. Agora ela está cursando MPhil/PhD na Universidade de Maurício em meio período e o projeto de pesquisa trata com o impacto das medidas de SPS sobre exportações de alimentos de Maurício. Seus interesses de pesquisa estão nas áreas de segurança de alimentos, microbiologia de alimentos, sistema de controle de alimentos, garantia de qualidade e comércio de alimentos. Ela também é uma conferencista em meio período na Universidade de Maurício e prestou serviços a módulos que pertencem à legislação de alimentos e à garantia da qualidade de alimentos. Ela também esteve envolvida em um Programa de Treinamento Regional SADC denominado "Fortalecimento do processamento e segurança de alimentos do setor informal" e desenvolveu um guia sobre processamento de alimentos. Ela também contribuiu para a redação de documentos e relatórios técnicos que pertencem aos sistemas de controle de alimentos e segurança de alimentos. Ela atualmente está envolvida no desenvolvimento de padrões de alimentos na Agência de Padrões de Maurício. 


\section{Daya Groburdhun}

Daya Goburdhun se associou à Faculdade de Agricultura em 1989 como conferencista e atualmente é Professora Associada na Food Science and Technology na Faculdade de Agricultura, Universidade de Maurício. O primeiro diploma de Daya foi na Agricultura e, posteriormente, especializou-se em Ciência dos Alimentos. Os interesses da pesquisa estão no sistema de controle de Alimentos; segurança de alimentos; tecnologia pós-colheita e nutrição. Os projetos de pesquisa financiados de Daya foram sobre a destoxificação de inibidores de proteases em pulsos; processamento de alimentos e tecnologia pós-colheita. Daya é um membro do Instituto de Tecnologia de Alimentos (E.U.A.). Daya participou do Programa de Treinamento Regional SADC "Fortalecimento do processamento e segurança dos alimentos do setor informal" e desenvolveu um guia sobre processamento de alimentos. Além de dar palestras para alunos graduados e pós-graduados nos últimos anos, Daya projetou diversos cursos sem distinção em Segurança de alimentos, qualidade de alimentos, alimentação saudável para empreendedores, crianças.. Daya também contribuiu para diversos trabalhos e relatórios técnicos relacionados à segurança de alimentos, qualidade, pós-colheita e nutrição. Daya está atualmente participando ativamente do desenvolvimento das normas de alimentos. 